\title{
Erratum: Asymmetric resonant exchange qubit under the influence of electrical noise [Phys. Rev. B 91, 235411 (2015)]
}

\author{
Maximilian Russ and Guido Burkard \\ (Received 10 December 2015; published 29 December 2015)
}

DOI: 10.1103/PhysRevB.92.239905

PACS number(s): 03.67.Lx, 73.21.La, 85.35.Be, 75.10.Jm, 99.10.Cd

We found that there are some mistakes in the logarithmic corrections in Eq. (20) and its derivation given in Appendix B of our paper. The correct expression for low-frequency noise is given by

$$
T_{\varphi}=\hbar\left[\frac{\omega_{\varepsilon}^{2}}{2} A_{\varepsilon} \log r+\frac{\omega_{\Delta}^{2}}{2} A_{\Delta} \log r+\frac{1}{4}\left(\omega_{\varepsilon, \varepsilon}^{2} A_{\varepsilon}^{2}+\omega_{\Delta, \Delta}^{2} A_{\Delta}^{2}+2 \omega_{\varepsilon, \Delta}^{2} A_{\varepsilon} A_{\Delta}\right) \log ^{2} r+\frac{1}{8} \omega_{\varepsilon, \varepsilon} \omega_{\Delta, \Delta} A_{\varepsilon} A_{\Delta}\right]^{-\frac{1}{2}} .
$$

We note that these corrections are very small (the missing square for the log in the third term, corresponding to the second line in the paper, is a typographical error); hence, all results of the paper including Fig. 6 are unchanged.

There is a typographical error on pages 3 and 4 where we introduce the expression for the longitudinal (transversal) correction of the Hamiltonian in Eq. (5) of the paper, $\delta \omega_{z(x)}$. The correct expressions are given without a factor of $1 / 2$,

$$
\delta \omega_{z}=-(J \delta J+3 j \delta j) / \omega, \quad \delta \omega_{x}=\sqrt{3}(J \delta j-j \delta J) / \omega .
$$

In Appendix B of the paper, in Eq. (B3), the higher-order corrections were not entirely correct. The expression should be

$$
\begin{aligned}
\log \left\langle e^{i \phi}\right\rangle= & -\frac{1}{2}\left(\left\langle\phi(t)^{2}\right\rangle-\langle\phi(t)\rangle^{2}\right) \\
= & -\frac{1}{2}\left(\left\langle\left[\int_{0}^{t} \omega_{\varepsilon} \delta \varepsilon\left(t^{\prime}\right) d t^{\prime}\right]^{2}\right\rangle+\left\langle\left[\int_{0}^{t} \omega_{\Delta} \delta \Delta\left(t^{\prime}\right) d t^{\prime}\right]^{2}\right\rangle+\frac{1}{4}\left\langle\left[\int_{0}^{t} \omega_{\varepsilon, \varepsilon} \delta \varepsilon\left(t^{\prime}\right)^{2} d t^{\prime}\right]^{2}\right\rangle+\frac{1}{4}\left\langle\left[\int_{0}^{t} \omega_{\Delta, \Delta} \delta \Delta\left(t^{\prime}\right)^{2} d t^{\prime}\right]^{2}\right\rangle\right. \\
& \left.+\left\langle\left[\int_{0}^{t} \omega_{\varepsilon, \Delta}^{2} \delta \varepsilon\left(t^{\prime}\right)^{2} \delta \Delta\left(t^{\prime}\right)^{2} d t^{\prime}\right]\right\rangle+\frac{1}{4}\left\langle\left[\int_{0}^{t} \omega_{\varepsilon, \varepsilon} \delta \varepsilon\left(t^{\prime}\right)^{2} d t^{\prime}\right]\left[\int_{0}^{t} \omega_{\Delta, \Delta} \delta \Delta\left(t^{\prime}\right)^{2} d t^{\prime}\right]\right\rangle\right) \\
& +\frac{1}{8}\left[\left\langle\int_{0}^{t} \omega_{\varepsilon, \varepsilon} \delta \varepsilon\left(t^{\prime}\right)^{2} d t^{\prime}\right\rangle^{2}+\left\langle\int_{0}^{t} \omega_{\Delta, \Delta} \delta \Delta\left(t^{\prime}\right)^{2} d t^{\prime}\right\rangle^{2}\right] .
\end{aligned}
$$

However, note that the main contribution to $\log \left\langle e^{i \phi}\right\rangle$, i.e., the first three terms in Eq. (B3), remain unchanged. Consequently, Eq. (B4) is given by

$$
\begin{aligned}
\log \left\langle e^{i \phi}\right\rangle= & -\frac{t^{2} \omega_{\varepsilon}^{2}}{2} \int_{-\infty}^{\infty} d \tilde{\omega} S_{\varepsilon}(\tilde{\omega}) \operatorname{sinc}^{2}\left(\frac{\tilde{\omega} t}{2}\right)-\frac{t^{2} \omega_{\Delta}^{2}}{2} \int_{-\infty}^{\infty} d \tilde{\omega} S_{\Delta}(\tilde{\omega}) \operatorname{sinc}^{2}\left(\frac{\tilde{\omega} t}{2}\right) \\
& -\frac{\omega_{\varepsilon, \varepsilon}^{2} t^{2}}{4}\left\{\iint_{-\infty}^{\infty} d \omega d \omega^{\prime} \operatorname{sinc}^{2}\left[\left(\frac{\omega+\omega^{\prime}}{2}\right) t\right] S_{\varepsilon}(\omega) S_{\varepsilon}\left(\omega^{\prime}\right)\right\}-\frac{\omega_{\varepsilon, \varepsilon} \omega_{\Delta, \Delta} t^{2}}{8}\left\langle\delta \varepsilon^{2}\right\rangle\left\langle\delta \Delta^{2}\right\rangle \\
& -\frac{\omega_{\Delta, \Delta}^{2} t^{2}}{4}\left\{\iint_{-\infty}^{\infty} d \omega d \omega^{\prime} \operatorname{sinc}^{2}\left[\left(\frac{\omega+\omega^{\prime}}{2}\right) t\right] S_{\Delta}(\omega) S_{\Delta}\left(\omega^{\prime}\right)\right\} \\
& -\frac{\omega_{\varepsilon, \Delta}^{2} t^{2}}{2}\left\{\iint_{-\infty}^{\infty} d \omega d \omega^{\prime} \operatorname{sinc}^{2}\left[\left(\frac{\omega+\omega^{\prime}}{2}\right) t\right] S_{\varepsilon}(\omega) S_{\Delta}\left(\omega^{\prime}\right)\right\} .
\end{aligned}
$$

Additionally, we noticed two typographical errors in the expression of the first-order noise terms. The parameter $A$ in Eqs. (B5) and (B6) should be omitted and in Eq. (B11) the leading factor $t^{2}$ needs to be deleted,

$$
\tilde{f}_{1}(t) \approx \exp \left\{-2 \omega_{q}^{2} A_{q}\left[k+\frac{t^{2}}{4} \log \left(\frac{\omega_{U}}{\omega_{R}}\right)\right]\right\} \propto \exp \left[-\omega_{q}^{2} A_{q} \frac{t^{2}}{2} \log \left(\frac{\omega_{U}}{\omega_{R}}\right)\right] .
$$

Moreover, the regime in which Eq. (B11) is valid should be corrected to $t \omega_{R} \ll 1$ and $t \omega_{U} \ll 1$.

Furthermore, in Eq. (B12), there is a missing factor $t$ in the sinc function,

$$
\iint_{-\infty}^{\infty} d \omega d \omega^{\prime} \operatorname{sinc}^{2}\left[\left(\frac{\omega+\omega^{\prime}}{2}\right) t\right] S_{\varepsilon, \Delta}(\omega) S_{\varepsilon, \Delta}\left(\omega^{\prime}\right)
$$

and the solution for the integral in Eq. (B13) is, in the general case, more complicated; hence, Eq. (B13) of the paper should not be considered. However, the final expression in Eq. (B14) is valid in the regime $t \omega_{R} \ll 1$ and $t \omega_{U} \ll 1$. 